\title{
A NOTE ON THE MATHEMATICAL FORMULATION OF THE PROBLEM OF NUMERICAL COORDINATE GENERATION*
}

\author{
BY \\ Z. U. A. WARSI \\ Mississippi State University
}

\begin{abstract}
A set of second order partial differential equations for the generation of three-dimensional grids around and between arbitrary shaped bodies has been proposed. These equations basically depend on the Gauss equations for a surface and have been structured in such a way that an automatic connection is established between the succeeding generated surfaces.

The vanishing of the Riemann curvature tensor has been used to isolate those fundamental equations which every coordinate system in either two- or three-dimensional Euclidean space must satisfy.
\end{abstract}

1. Introduction. The problem of generating spatial coordinates by numerical methods is a problem of much interest in practically all branches of engineering science and physics. At present a number of techniques are under active development for the generation of two- and three-dimensional coordinates around and between bodies of arbitrary shapes. Among these efforts two easily discernable groups can be formed: (i) the methods based on the solution of certain PDE's, preferably of the elliptic type, and (ii) the algebraic methods. In a large number of methods in the first group a set of inhomogeneous Laplace equations is taken as the basic generating system. These equations are then inverted and solved for the Cartesian coordinates in the transformed plane. Based on this line of approach which started with the work of Winslow [1] some very practical results, particularly in two dimensions, have been obtained by Thompson et al. [2] and others. ${ }^{1}$ For the generation of plane curvilinear coordinates some authors have also used hyperbolic and parabolic systems of equations, [3]. For the methods in the second group, i.e., the algebraic methods, refer to [3].

In this paper we have first considered the formulation of a $3 D$ grid generation scheme which is basically dependent on the Gauss' equations for a surface. In this scheme a series

\footnotetext{
* Received May 10, 1982. This research has been supported by the Air Force Office of Scientific Research, under grant AFOSR 80-0185. The material of this paper is based on an extended paper submitted at a NASA-AFOSR sponsored conference on numerical grid generation held at Nashville, Tennessee April 13-16, 1982, [6].

${ }^{1}$ Refer to [3] for an extensive bibliography on the subject.
} 
of surfaces are generated based on the given data of arbitrary shaped inner and outer surfaces. The method has also been structured in such a way that the variation of the third coordinate from one generated surface to the next is fully reflected in the system of generating equations. It has been found that these generating system of equations are simple to implement numerically. In particular, the solution of the proposed equations tends to the solution of the Laplace equations in the transformed plane in case the surface becomes a Cartesian plane. An exact solution of these equations for the case of three-dimensional curvilinear coordinates between a prolate ellipsoid and a sphere has been obtained.

In a plane, or a surface, or a $3 D$ space there are endless possibilities of introducing either orthogonal or non-orthogonal coordinates. This realization imparts a sense of arbitrariness to the choice of the method to be used for coordinate generation. If it is $a$ priori decided that the method should be based on solving partial differential equations then the arbitrariness in the selection of the appropriate equations for the generation of coordinates becomes a problem to be resolved. In Sec. 3 of this paper it has been shown that despite this arbitrariness it is possible to uncover certain sets of equations which must invariably be satisfied no matter which equations or methods have been used to generate the coordinates. For a detailed discussion of the methods discussed here and on some numerical results refer to Warsi [4-7].

2. Generating system based on the Gauss' equations. In the process of formulation of a $3 D$ coordinate generation problem it is helpful to imagine the coordinates of a point in space as the intersection of three distinct surfaces on each of which one coordinate is held fixed. Using the convention of a right-handed curvilinear coordinate system $x^{1}, x^{2}, x^{3}$ or $\xi, \eta, \zeta$ (refer to Fig. 1) we introduce the coordinates in the surface $x^{\nu}=$ const. through the following scheme.

$$
\nu=1:\left(x^{2}, x^{3}\right) ; \quad \nu=2:\left(x^{3}, x^{1}\right) ; \quad \nu=3:\left(x^{1}, x^{2}\right) .
$$

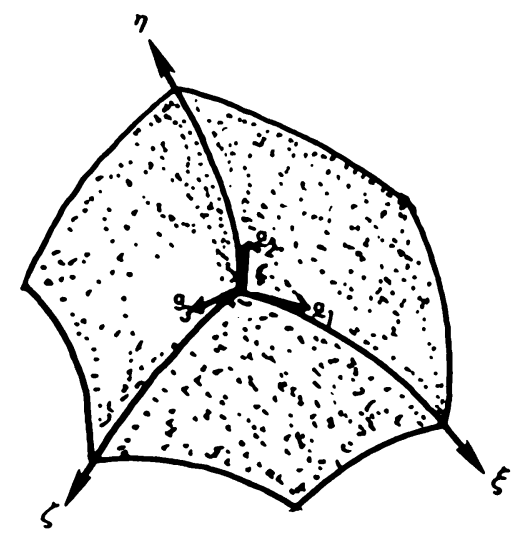

FIG. 1. Right-handed coordinate arrangement and basis vectors. 
Thus the unit normal vector on the surface $x^{\nu}=$ const. is

$$
\mathbf{n}^{(\nu)}=\left(\mathbf{r}_{\alpha} \times \mathbf{r}_{\beta}\right) /\left|\mathbf{r}_{\alpha} \times \mathbf{r}_{\beta}\right|
$$

where

$$
\nu=1: \alpha=2, \beta=3 ; \quad \nu=2: \alpha=3, \beta=1 ; \quad \nu=3: \alpha=1, \beta=2 .
$$

From elementary differential geometry [8] we have the result that the rectangular Cartesian coordinates $\mathbf{r}=(x, y, z)$ or $\left(x_{1}, x_{2}, x_{3}\right)$ of any point on every surface embedded in an Euclidean $E_{3}$ must satisfy the equations of Gauss. The Gauss equations for a surface $x^{\nu}=$ const. are given by

$$
\mathbf{r}_{\alpha \beta}=\Upsilon_{\alpha \beta}^{\delta} \mathbf{r}_{\delta}+b_{\alpha \beta} \mathbf{n}^{(\nu)},
$$

where all the Greek indices except $\nu$ can assume only two values. The values of $\alpha, \beta$ and the range of $\delta$ with $\nu$ follow the scheme given in (2). In Eq. (3),

$$
\mathbf{r}_{\delta}=\frac{\partial \mathbf{r}}{\partial x^{\delta}}, \quad \mathbf{r}_{\alpha \beta}=\frac{\partial^{2} \mathbf{r}}{\partial x^{\alpha} \partial x^{\beta}},
$$

$\Upsilon_{\alpha \beta}^{\delta}$ are the surface Christoffel symbols of the second kind, ${ }^{2}$ i.e.,

$$
\begin{aligned}
\Upsilon_{\alpha \beta}^{\delta} & =g^{\sigma \delta}[\alpha \beta, \sigma], \\
{[\alpha \beta, \sigma] } & =\frac{1}{2}\left(\frac{\partial g_{\alpha \sigma}}{\partial x^{\beta}}+\frac{\partial g_{\beta \sigma}}{\partial x^{\alpha}}-\frac{\partial g_{\alpha \beta}}{\partial x^{\sigma}}\right),
\end{aligned}
$$

and $b_{\alpha \beta}$ are the coefficients of the second fundamental form. Since on the surface $x^{\nu}=$ const. the vector $\mathbf{n}^{(\nu)}$ is orthogonal to the surface vectors $\mathbf{r}_{\delta}$,

$$
b_{\alpha \beta}=\mathbf{n}^{(\nu)} \cdot \mathbf{r}_{\alpha \beta} \text {. }
$$

For the purpose of a clear notation we denote the space Christoffel symbols as

$$
\Gamma_{i j}^{l}=g^{k l}[i j, k] \text {, }
$$

where

$$
[i j, k]=\frac{1}{2}\left(\frac{\partial g_{i k}}{\partial x^{j}}+\frac{\partial g_{j k}}{\partial x^{i}}-\frac{\partial g_{i j}}{\partial x^{k}}\right)
$$

Using (6a), we have

$$
\mathbf{r}_{i j}=\frac{\partial^{2} \mathbf{r}}{\partial x^{i} \partial x^{j}}=\Gamma_{i j}^{l} \mathbf{r}_{l},
$$

where all the Latin indices assume three values.

To fix ideas, we envisage a surface which is formed of the surface coordinates $(\xi, \eta)$ and on which $\zeta=$ const. Dropping the index $\nu$ in Eq. (3), the three equations for the second derivatives of the Cartesian coordinates are

$$
\mathbf{r}_{\xi \xi}=\Upsilon_{11}^{\delta} \mathbf{r}_{\delta}+b_{11} \mathbf{n}, \quad \mathbf{r}_{\xi \eta}=\Upsilon_{12}^{\delta} \mathbf{r}_{\delta}+b_{12} \mathbf{n}, \quad \mathbf{r}_{\eta \eta}=\Upsilon_{22}^{\delta} \mathbf{r}_{\delta}+b_{22} \mathbf{n}
$$

In Eqs. (8) $\mathbf{n}$ is orthogonal to both $\mathbf{r}_{\boldsymbol{\xi}}$ and $\mathbf{r}_{\eta}$ and the coefficients of the first fundamental

\footnotetext{
${ }^{2}$ Refer to Appendix A for a collection of other formulae.
} 
form $g_{11}, g_{12}, g_{22}$ are assumed to have been evaluated at $\zeta=$ const. Obviously

$$
g_{11}=x_{\xi}^{2}+y_{\xi}^{2}+z_{\xi}^{2}, \quad g_{12}=x_{\xi} x_{\eta}+y_{\xi} y_{\eta}+z_{\xi} z_{\eta}, \quad g_{22}=x_{\eta}^{2}+y_{\eta}^{2}+z_{\eta}^{2} .
$$

Multiplying Eq. (8a) by $g_{22}$, Eq. (8b) by $-2 g_{12}$, Eq. (8c) by $g_{11}$ and adding the three equations, we get

$$
\mathfrak{f r}+\left[\left(\Delta_{2} \xi\right) \mathbf{r}_{\xi}+\left(\Delta_{2} \eta\right) \mathbf{r}_{\eta}\right] G_{3}=\mathbf{n} R
$$

where

$$
\begin{aligned}
£ & =g_{22} \partial_{\xi \xi}-2 g_{12} \partial_{\xi \eta}+g_{11} \partial_{\eta \eta}, \\
\Delta_{2} & =\left[\partial_{\xi}\left\{\left(g_{22} \partial_{\xi}-g_{12} \partial_{\eta}\right) / \sqrt{G_{3}}\right\}+\partial_{\eta}\left\{\left(g_{11} \partial_{\eta}-g_{12} \partial_{\xi}\right) / \sqrt{G_{3}}\right\}\right] / \sqrt{G_{3}}, \\
G_{3} & =g_{11} g_{22}-\left(g_{12}\right)^{2} \\
\mathbf{n} & =(X, Y, Z), \\
X & =\left(y_{\xi} z_{\eta}-y_{\eta} z_{\xi}\right) / \sqrt{G_{3}}, \quad Y=\left(x_{\eta} z_{\xi}-x_{\xi} z_{\eta}\right) / \sqrt{G_{3}}, \quad Z=\left(x_{\xi} y_{\eta}-x_{\eta} y_{\xi}\right) / \sqrt{G_{3}}, \\
R & =\left(g_{22} b_{11}-2 g_{12} b_{12}+g_{11} b_{22}\right)=G_{3}\left(k_{1}+k_{2}\right),
\end{aligned}
$$

where $k_{1}+k_{2}$ is twice the mean curvature of the surface.

The operator $\Delta_{2}^{(3)}$ or simply $\Delta_{2}$ defined in (11b) is the second order differential operator of Beltrami [8], for the surface $\zeta=$ const. For any surface $x^{\nu}=$ const. and following the scheme (2), we have

$$
\Delta_{2}^{(\nu)}=\left[\partial_{\alpha}\left\{\left(g_{\beta \beta} \partial_{\alpha}-g_{\alpha \beta} \partial_{\beta}\right) / \sqrt{G_{\nu}}\right\}+\partial_{\beta}\left\{\left(g_{\alpha \alpha} \partial_{\beta}-g_{\alpha \beta} \partial_{\alpha}\right) / \sqrt{G_{\nu}}\right\}\right] / \sqrt{G_{\nu}},
$$

where $G_{\nu}$ are defined in Eq. (A.9). It is easy to show by using the surface Christoffel symbols $\Upsilon_{\alpha \beta}^{\delta}$ that

$$
\begin{aligned}
& \Delta_{2} \xi=\left(2 g_{12} \Upsilon_{12}^{1}-g_{22} \Upsilon_{11}^{1}-g_{11} \Upsilon_{22}^{1}\right) / G_{3}, \\
& \Delta_{2} \eta=\left(2 g_{12} \Upsilon_{12}^{2}-g_{22} \Upsilon_{11}^{2}-g_{11} \Upsilon_{22}^{2}\right) / G_{3},
\end{aligned}
$$

where the metric coefficients $g_{\alpha \beta}$ are those as defined in (9). It is interesting to see that when the Laplacian operator $\nabla^{2}$ for a two-dimensional Cartesian space is applied to the curvilinear coordinates $(\xi, \eta)$ in an Euclidean plane, the resulting expressions are exactly of the same form as (13a, b), that is (refer to Eq. (A.13)),

$$
\begin{aligned}
\nabla^{2} \xi & =\left(2 g_{12} \Gamma_{12}^{1}-g_{22} \Gamma_{11}^{1}-g_{11} \Gamma_{22}^{1}\right) /(J)^{2}, \\
\nabla^{2} \eta & =\left(2 g_{12} \Gamma_{12}^{2}-g_{22} \Gamma_{11}^{2}-g_{11} \Gamma_{22}^{2}\right) /(J)^{2},
\end{aligned}
$$

where now

$$
g_{11}=x_{\xi}^{2}+y_{\xi}^{2}, \quad g_{12}=x_{\xi} x_{\eta}+y_{\xi} y_{\eta}, \quad g_{22}=x_{\eta}^{2}+y_{\eta}^{2}, \quad J=x_{\xi} y_{\eta}-x_{\eta} y_{\xi} .
$$

Though the right-hand side term $R$ defined in (11e) has the necessary extrinsic effects, nevertheless we must have an explicit dependence of $\mathbf{r}=(x, y, z)$ on the third coordinate $\zeta$. Thus using Eq. (A.11) we have 


$$
\begin{aligned}
& \mathbf{r}_{\xi \xi}=\Gamma_{11}^{1} \mathbf{r}_{\xi}+\Gamma_{11}^{2} \mathbf{r}_{\eta}+\Gamma_{11}^{3} \mathbf{r}_{\zeta}, \\
& \mathbf{r}_{\xi \eta}=\Gamma_{12}^{1} \mathbf{r}_{\xi}+\Gamma_{12}^{2} \mathbf{r}_{\eta}+\Gamma_{12}^{3} \mathbf{r}_{\zeta}, \\
& \mathbf{r}_{\eta \eta}=\Gamma_{22}^{1} \mathbf{r}_{\xi}+\Gamma_{22}^{2} \mathbf{r}_{\eta}+\Gamma_{22}^{3} \mathbf{r}_{\zeta},
\end{aligned}
$$

and we evaluate each of these derivatives at $\zeta=$ const. Taking the dot product of Eqs. (15) with $\mathbf{n}$ and comparing with Eqs. (5), we get

$$
b_{11}=\lambda \Gamma_{11}^{3}, \quad b_{12}=\lambda \Gamma_{12}^{3}, \quad b_{22}=\lambda \Gamma_{22}^{3},
$$

where

$$
\lambda=\mathbf{n} \cdot \mathbf{r}_{\zeta}=X x_{\zeta}+Y y_{\zeta}+Z z_{\zeta} .
$$

Thus, the expression (11e) for $R$ is replaced by

$$
R=\lambda\left[g_{11} \Gamma_{22}^{3}-2 g_{12} \Gamma_{12}^{3}+g_{22} \Gamma_{11}^{3}\right] .
$$

2.1 Fundamental generating system of equations. We now impose the following differential constraints on the coordinates $\xi$ and $\eta$ :

$$
\Delta_{2} \xi=0, \quad \Delta_{2} \eta=0,
$$

and take them as the basic generating equations for the coordinates in a surface. A comparison of Eqs. (13) and (14) has already shown that $\Delta_{2}$ is not a $2 D$ Laplace operator except when the surface degenerates into a plane having no dependence on the $z$-coordinate.

It is a well-known result in differential geometry that the isothermic coordinates in a surface satisfy Eqs. (18) identically. The isothermic coordinates $\xi$ and $\eta$ are those orthogonal coordinates in a surface which yield $g_{22}=g_{11}$. The situation here is parallel to the choice of the Laplace equations $\nabla^{2} \xi=0, \nabla^{2} \eta=0$ for the generation of plane curvilinear coordinates [2], which are also satisfied by the conformal coordinates in a plane. The important point to note here is that the satisfaction of the Laplace equations is a necessary but not a sufficient condition for the existence of conformal coordinates. Similarly, the satisfaction of equations (18) is a necessary but not a sufficient condition for the existence of isothermic coordinates. It would, therefore, be more meaningful if we interpret Eqs. (18) as providing a set of differential constraints ${ }^{3}$ on the metric coefficients $g_{11}, g_{12}$, and $g_{22}$ defined in (9).

Having chosen Eqs. (18) as the generating system, the equations for the determination of the Cartesian coordinates, viz., Eq. (10) becomes

$$
\mathrm{fr}=\mathrm{n} R,
$$

where $£, \mathbf{n}$ and $R$ have been defined in (11a), (11d), and (17) respectively. The three scalar equations in expanded form are now

$$
\begin{gathered}
g_{22} x_{\xi \xi}-2 g_{12} x_{\xi \eta}+g_{11} x_{\eta \eta}=X R, \\
g_{22} y_{\xi \xi}-2 g_{12} y_{\xi \eta}+g_{11} y_{\eta \eta}=Y R,
\end{gathered}
$$

\footnotetext{
${ }^{3} \mathrm{~A}$ manifestation of the many possibilities for introducing coordinates in a given place.
} 


$$
g_{22} z_{\xi \xi}-2 g_{12} z_{\xi \eta}+g_{11} z_{\eta \eta}=Z R
$$

For a plane $z=$ const., $R=0$ and the Eqs. (20) are the inversions of the Laplace equations in the $\xi \eta$-plane.

It can be shown that Eqs. (20) can be combined to obtain the equations of a surface $z=z(x, y)$ in the well known form,

$$
\alpha z_{x x}-2 \beta z_{x y}+\gamma z_{y y}=2 H M
$$

where

$$
\begin{aligned}
2 H & =k_{1}+k_{2}=R / G_{3}, \quad M=1+p^{2}+q^{2}, \quad p=z_{x}, q=z_{y}, \\
\alpha & =\left(1+q^{2}\right) / \sqrt{M}, \quad \beta=p q / \sqrt{M}, \quad \gamma=\left(1+p^{2}\right) / \sqrt{M} .
\end{aligned}
$$

Using the following definitions and identities

$$
\begin{aligned}
G_{3} & =g_{11} g_{22}-\left(g_{12}\right)^{2}, \quad X=-p / \sqrt{M}, \quad Y=-q / \sqrt{M}, \quad Z=1 / \sqrt{M}, \\
\Delta_{1}(x, x) & =\left(1-X^{2}\right) G_{3}, \quad \Delta_{1}(x, y)=-X Y G_{3}, \quad \Delta_{1}(y, y)=\left(1-Y^{2}\right) G_{3},
\end{aligned}
$$

where

$$
\Delta_{1}(a, b)=g_{22} a_{\xi} b_{\xi}-g_{12}\left(a_{\xi} b_{\eta}+a_{\eta} b_{\xi}\right)+g_{11} a_{\eta} b_{\eta},
$$

calculating $z_{\xi \xi}, z_{\xi \eta}, z_{\eta \eta}$ from $z_{\xi}, z_{\eta}$ and substituting these expressions in (20c) while using Eqs.(20a, b), we get Eq. (21).

We now use the result that if $(\xi, \eta)$ is a permissible system of coordinates in a surface then so is $(\bar{\xi}, \bar{\eta})$, where $\bar{\xi}=\bar{\xi}(\xi, \eta), \bar{\eta}=\bar{\eta}(\xi, \eta)$, provided that the Jacobian of the transformation does not vanish. It is a straight forward matter to show that on coordinate transformation, Eqs. (20) become

$$
\overline{\mathfrak{f}} x=\bar{X} \bar{R}, \quad \overline{\mathfrak{f}} y=\bar{Y} \bar{R}, \quad \overline{\mathfrak{f}} z=\bar{Z} \bar{R},
$$

where

$$
\begin{aligned}
\bar{£} & =\bar{g}_{22} \partial_{\bar{\xi} \bar{\xi}}-2 \bar{g}_{12} \partial_{\bar{\xi} \bar{\eta}}+\bar{g}_{11} \partial_{\bar{\eta} \eta}+\bar{P}_{\bar{\xi}}+\bar{Q}_{\bar{\eta}}, \\
\bar{P} & =\bar{g}_{22} P_{11}^{1}-2 \bar{g}_{12} P_{12}^{1}+\bar{g}_{11} P_{22}^{1}, \\
\bar{Q} & =\bar{g}_{22} P_{11}^{2}-2 \bar{g}_{12} P_{12}^{2}+\bar{g}_{11} P_{22}^{2}, \\
P_{\mu \sigma}^{\gamma} & =\frac{\partial x^{\alpha}}{\partial \bar{x}^{\mu}} \frac{\partial x^{\beta}}{\partial \bar{x}^{\sigma}} \frac{\partial^{2} \bar{x}^{\gamma}}{\partial x^{\alpha} \partial x^{\beta}},
\end{aligned}
$$

and $\bar{X}, \bar{Y}, \bar{Z}$, and $\bar{R}$ are exactly the same expressions as given in (11d) and (17), in the $\bar{x}^{\alpha}$ coordinate system. It is preferable to solve Eqs. (22) with $P_{\beta \delta}^{\alpha}$ as arbitrarily prescribed functions of the coordinates. This aspect of the formulation therefore provides a capability to redistribute the coordinate systems in the surface in any desired manner. ${ }^{4}$

2.2 Example of a solution algorithm. The discussion that follows pertains to the case when it is desired to generate the $3 D$ curvilinear coordinates between two arbitrary shaped smooth surfaces. Let the surface coordinates $(\zeta, \xi)$ of the inner body $\eta=\eta_{B}$ and of the

\footnotetext{
${ }^{4}$ For a limiting form of Eqs. (22) refer to Appendix B.
} 
outer body $\eta=\eta_{\infty}$ be the same coordinates. Thus

$$
\eta=\eta_{B}: \mathbf{r}=\mathbf{r}_{B}(\xi, \zeta) ; \quad \eta=\eta_{\infty}: \mathbf{r}=\mathbf{r}_{\infty}(\xi, \zeta)
$$

are known functions (either analytically or numerically) and hence the needed partial derivatives with respect to $\xi$ and $\zeta$ are directly available at the surfaces.

For the computation of $\mathbf{r}_{\zeta}$ in the field one must first note that the coordinate $\zeta$ may not, in general, satisfy the equation $\Delta_{2}^{(2)} \zeta=0$. Consequently, $\mathbf{r}_{\zeta}$ must satisfy the equation

$$
\mathfrak{E}^{(2)} \mathbf{r}+G_{2}\left(\Delta_{2}^{(2)} \zeta\right) \mathbf{r}_{\zeta}=G_{2}\left(k_{1}^{(2)}+k_{2}^{(2)}\right) \mathbf{n}^{(2)} .
$$

From this equation we devise a weighted integral formula ${ }^{5}$

$$
\mathbf{r}_{\zeta}=\int\left[f_{1}(\eta)\left(\mathbf{r}_{\zeta \zeta}\right)_{B}+f_{2}(\eta)\left(\mathbf{r}_{\zeta \zeta}\right)_{\infty}\right] d \zeta
$$

where

$$
\begin{aligned}
\left(\mathbf{r}_{\zeta \zeta}\right)_{B, \infty}= & {\left[\frac{G_{2}}{g_{11}}\left(k_{1}^{(2)}+k_{2}^{(2)}\right) \mathbf{n}^{(2)}+\frac{2 g_{13}}{g_{11}} \mathbf{r}_{\xi \zeta}-\frac{g_{33}}{g_{11}} \mathbf{r}_{\xi \xi}\right.} \\
& \left.-\frac{\sqrt{G_{2}}}{g_{11}}\left\{\frac{\partial}{\partial \zeta}\left(\frac{g_{11}}{\sqrt{G_{2}}}\right)-\frac{\partial}{\partial \xi}\left(\frac{g_{13}}{\sqrt{G_{2}}}\right)\right\}_{\zeta}\right]_{B, \infty},
\end{aligned}
$$

and

$$
f_{1}\left(\eta_{B}\right)=1, \quad f_{1}\left(\eta_{\infty}\right)=0, \quad f_{2}\left(\eta_{B}\right)=0, \quad f_{2}\left(\eta_{\infty}\right)=1
$$

There is no difficulty in the numerical evaluation of (25a) in an iterative cycle after the weighting functions $f_{1}$ and $f_{2}$ have been prescribed a priori.

Referring to Fig. (2a), we now solve Eqs. (20) or (22) for each $\zeta=$ const., by prescribing the values of $x, y, z$ on the curves $C_{1}$ and $C_{2}$ which respectively represent the curves on $B$ and $\infty$. In Fig. (2b) $C_{3}$ and $C_{4}$ are the cut lines on which periodic conditions are to be imposed.

2.3 An exact solution of the proposed equations. The following example demonstrates that the proposed set of generating equations (22) are consistent and provide nontrivial solutions.

We consider the case of coordinate generation between an inner body $\eta=\eta_{B}$ which is a prolate ellipsoid and an outer boundary $\eta=\eta_{\infty}$ which is a sphere. The coordinates which vary on these two surfaces are $\xi$ and $\zeta$. A curve $C_{1}$ on the inner surface for $\zeta=$ const. is

$$
x=\tau \cosh \eta_{B} \cos \zeta, \quad y=\tau \sinh \eta_{B} \sin \zeta \cos \xi, \quad z=\tau \sinh \eta_{B} \sin \zeta \sin \xi,
$$

where $\tau$ and $\eta_{B}$ are the parameters of the ellipsoid. Similarly the curve $C_{2}$ corresponding to the same $\zeta=$ const. on the outer surface is

$$
x=\exp \left(\eta_{\infty}\right) \cos \zeta, \quad y=\exp \left(\eta_{\infty}\right) \sin \zeta \cos \xi, \quad z=\exp \left(\eta_{\infty}\right) \sin \zeta \sin \xi .
$$

\footnotetext{
${ }^{5}$ The discussion given here is directed to the situation of Fig. (2a). For other situations, e.g., simply-connected domains or multibody problems the method of calculating $\mathbf{r}_{\zeta}$ must always be devised separately. Note also that Eq. (24) reflects the condition $\Delta_{2}^{(2)} \xi=0$.
} 


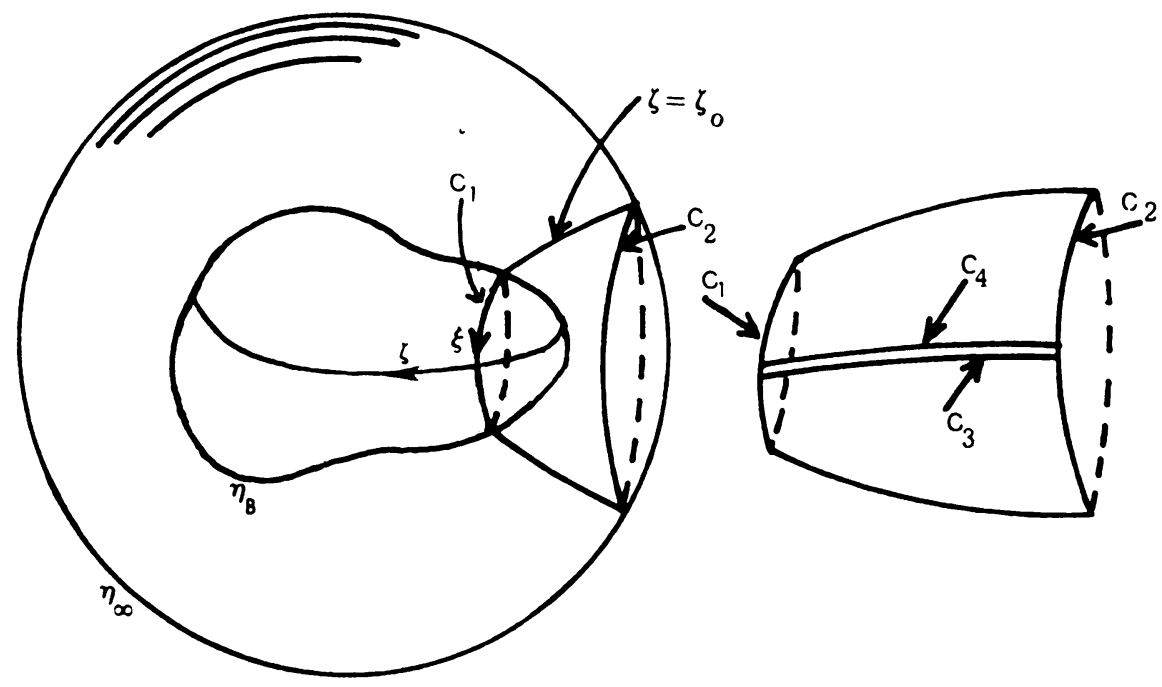

Fig. 2. (a) Topology of the given surfaces.

(b) Surfaces to be generated.

In order to generate surfaces on which $\xi$ and $\eta$ are the coordinates and in which the $\eta$-coordinate can nonuniformly be distributed (contraction or expansion in the $\eta$ coordinates), we assume

$$
\xi=\xi(\bar{\xi}), \quad \eta=\eta(\bar{\eta})+\eta_{B},
$$

where $\bar{\xi}=0$ corresponding to $\xi=0$ and $\bar{\eta}=\bar{\eta}_{B}$ corresponding to $\eta=\eta_{B}$. Thus $\xi(0)=0$, $\eta\left(\bar{\eta}_{B}\right)=0$. Under the transformation (27), the only nonzero components of $P_{\mu \sigma}^{\gamma}$ are $P_{11}^{1}$ and $P_{22}^{2}$. Writing

$$
\lambda(\bar{\xi})=\frac{d \xi}{d \bar{\xi}}, \quad \theta(\bar{\eta})=\frac{d \eta}{d \bar{\eta}}
$$

we have

$$
P_{11}^{1}=-\frac{1}{\lambda} \frac{d \lambda}{d \bar{\xi}}, \quad P_{22}^{2}=-\frac{1}{\theta} \frac{d \theta}{d \bar{\eta}}
$$

Based on the forms of the boundary conditions (26a, b) we assume the following forms for $x, y, z$ for each $\zeta=$ const.:

$$
x=f(\bar{\eta}) \cos \zeta, \quad y=\phi(\bar{\eta}) \sin \zeta \cos \xi, \quad z=\phi(\bar{\eta}) \sin \zeta \sin \xi
$$

The boundary conditions are

$$
\begin{array}{ll}
f\left(\bar{\eta}_{B}\right)=\tau \cosh \eta_{B}, & f\left(\bar{\eta}_{\infty}\right)=\exp \left(\eta_{\infty}\right), \\
\phi\left(\bar{\eta}_{B}\right)=\tau \sinh \eta_{B}, & \phi\left(\bar{\eta}_{\infty}\right)=\exp \left(\eta_{\infty}\right) .
\end{array}
$$

Using the expressions of (29) we calculate the various partial derivatives, metric coefficients, and all other data as needed for Eqs. (22). On substitution we get an equation containing $\sin ^{2} \zeta$ and $\cos ^{2} \zeta$. Equating to zero the coefficients of $\sin ^{2} \zeta$ and $\cos ^{2} \zeta$, we obtain 


$$
\begin{aligned}
f^{\prime \prime} / f^{\prime} & =\theta^{\prime} / \theta+\phi^{\prime} / \phi, \\
\phi^{\prime \prime} / \phi^{\prime} & =\theta^{\prime} / \theta+\phi^{\prime} / \phi,
\end{aligned}
$$

where a prime denotes differentiation with respect to $\bar{\eta}$. On direct integrations of Eqs. (31) and (32) under the boundary conditions (30), we get

$$
\begin{aligned}
f(\bar{\eta}) & =A \exp (B \eta(\bar{\eta}))+C, \\
\phi(\bar{\eta}) & =D \exp (B \eta(\bar{\eta})),
\end{aligned}
$$

where

$$
\begin{aligned}
& A=\tau\left[\left(\exp \left(\eta_{\infty}\right)-\tau \cosh \eta_{B}\right) \sinh \eta_{B}\right] /\left(\exp \left(\eta_{\infty}\right)-\tau \sinh \eta_{B}\right), \\
& B=\left(\eta_{\infty}-\ln \left(\tau \sinh \eta_{B}\right)\right) /\left(\eta_{\infty}-\eta_{B}\right), \\
& C=\tau\left[\exp \left(\eta_{\infty}\right)\left(\cosh \eta_{B}-\sinh \eta_{B}\right)\right] /\left(\exp \left(\eta_{\infty}\right)-\tau \sinh \eta_{B}\right), \\
& D=\tau \sinh \eta_{B} .
\end{aligned}
$$

As an application, we take

$$
\xi(\bar{\xi})=a \bar{\xi}, \quad \eta(\bar{\eta})=b\left(\bar{\eta}-\bar{\eta}_{B}\right) k^{\bar{\eta}}
$$

where $a, b$, and $k$ are constants. Thus

$$
\eta(\bar{\eta})=\frac{\left(\eta_{\infty}-\eta_{B}\right)\left(\bar{\eta}-\bar{\eta}_{B}\right)}{\bar{\eta}_{\infty}-\bar{\eta}_{B}} k^{\left(\bar{\eta}-\bar{\eta}_{\infty}\right)} .
$$

By taking a value of $k$ slightly greater than one $(k=1.05)$ we can have sufficient contraction in the $\bar{\eta}$-coordinate near the inner surface. For the chosen problem since the dependence on $\zeta$ is simple, we find that the generated coordinates between a prolate ellipsoid and a sphere are

$$
\begin{gathered}
x=[A \exp (B \eta(\bar{\eta}))+C] \cos \zeta, \quad y=D \exp (B \eta(\bar{\eta})) \sin \zeta \cos \xi, \\
z=D \exp (B \eta(\bar{\eta})) \sin \zeta \sin \xi .
\end{gathered}
$$

This example also shows that the chosen generating system of equations (20) or (22) are capable of providing non-isothermic coordinates between smooth surfaces.

3. Differential equations based on the Riemann tensor. In any given space there are endless possibilities for the introduction of coordinate curves. Each chosen set of curves determines its own metric components. For example, in a Cartesian plane besides introducing rectangular Cartesian coordinates $x, y$, we also have endless possibilities for introducing either orthogonal or nonorthogonal coordinate curves. However, as is well known, there is a basic differential constraint on the variations of $g_{i j}$ 's irrespective of the coordinate system. Since the curvature of an Euclidean two-dimensional plane is identically zero, the basic differential constraint on the $g_{i j}$ 's is

$$
\left(G_{3}\right)^{-1 / 2} R_{1212}=\frac{\partial}{\partial \eta}\left(\frac{\sqrt{G_{3}}}{g_{11}} \Gamma_{11}^{2}\right)-\frac{\partial}{\partial \xi}\left(\frac{\sqrt{G_{3}}}{g_{11}} \Gamma_{12}^{2}\right)=0,
$$

where $\xi, \eta$ are any arbitrary coordinate curves in the plane. Thus no matter which coordinate system is introduced in a plane, the corresponding matrics $g_{i j}$ must satisfy Eq. 
(37). Equation (37) has also been used as the basic generating equation for the generation of orthogonal coordinates in a plane [9]. In general, the Riemann curvature tensor $R_{r j n}$ defined as,

$$
\begin{aligned}
R_{r j n p}= & \frac{1}{2}\left(\frac{\partial^{2} g_{r p}}{\partial x^{j} \partial x^{n}}+\frac{\partial^{2} g_{j n}}{\partial x^{r} \partial x^{p}}-\frac{\partial^{2} g_{r n}}{\partial x^{j} \partial x^{p}}-\frac{\partial^{2} g_{j p}}{\partial x^{r} \partial x^{n}}\right) \\
& +g^{t s}([j n, s][r p, t]-[j p, s][r n, t])
\end{aligned}
$$

defines the components of the curvature tensor of any general space. If the space is $N$-dimensional, then the number of components $R_{r j n p}$ are given by $N^{2}\left(N^{2}-1\right) / 12$. Thus for $N=2$ there is one distinct surviving component stated in Eq. (37). However, for $N=3$, it has six distinct components $R_{1212}, R_{1313}, R_{2323}, R_{1213}, R_{1232}, R_{1323}$. If the $3 D$-space is Euclidean, then its curvature is zero, so that the six equations

$$
\begin{array}{lll}
R_{1212}=0, & R_{1313}=0, & R_{2323}=0, \\
R_{1213}=0, & R_{1232}=0, & R_{1323}=0
\end{array}
$$

determine the differential constraints for the six metric coefficients $g_{i j}$ in any coordinate system introduced in an Euclidean space. These equations in the expanded form have been given in [5] and [6].

Equations (39) are those consistent set of partial differential equations which must always be satisfied by the metric coefficients $g_{i j}$. In the $3 D$ case Eqs. (39) are six equations in six unknowns, and, therefore, they form a closed system of equations. In contrast, for the $2 D$ case there is only one equation (Eq. (37)) and three unknowns $g_{11}, g_{12}, g_{22}$ and therefore some constraints are needed to turn Eq. (37) (such as orthogonality [9]) into a solvable equation.

To obtain the Cartesian coordinates on the basis of the available $g_{i j}$ 's, we introduce the unit base vectors $\boldsymbol{\lambda}_{i}$ as

$$
\boldsymbol{\lambda}_{i}=\mathbf{a}_{i} / \sqrt{g_{i i}}, \quad \text { no sum on } i .
$$

Let the components of $\lambda_{i}$ along the rectangular Cartesian axes be denoted as $u_{i}, v_{i}, w_{i}$, so that

$$
\lambda_{i}=\left(u_{i}, v_{i}, w_{i}\right)
$$

where

$$
\begin{array}{lll}
u_{1}=x_{\xi} / \sqrt{g_{11}}, & v_{1}=y_{\xi} / \sqrt{g_{11}}, & w_{1}=z_{\xi} / \sqrt{g_{11}}, \\
u_{2}=x_{\eta} / \sqrt{g_{22}}, & v_{2}=y_{\eta} / \sqrt{g_{22}}, & w_{2}=z_{\eta} / \sqrt{g_{22}}, \\
u_{3}=x_{\zeta} / \sqrt{g_{33}}, & v_{3}=y_{\zeta} / \sqrt{g_{22}}, & w_{3}=z_{\zeta} / \sqrt{g_{33}} .
\end{array}
$$

If the components $u_{i}, v_{i}, w_{i}$ become known by some method then it is possible to evaluate the Cartesian coordinates through the line integrals

$$
\mathbf{r}=\int\left(\boldsymbol{\lambda}_{1} \sqrt{g_{11}} d \xi+\lambda_{2} \sqrt{g_{22}} d \eta+\lambda_{3} \sqrt{g_{33}} d \zeta\right) .
$$


The determination of $u_{i}, v_{i}, w_{i}$ is a separate problem which we now consider. First of all using (40) in Eq. (A.11), we get a system of first order partial differential equations

$$
\begin{aligned}
\frac{\partial \lambda_{i}}{\partial x^{j}}= & \lambda_{1}\left(\frac{g_{11}}{g_{i i}}\right)^{1 / 2} \Gamma_{i j}^{1}+\lambda_{2}\left(\frac{g_{22}}{g_{i i}}\right)^{1 / 2} \Gamma_{i j}^{2} \\
& +\lambda_{3}\left(\frac{g_{33}}{g_{i i}}\right)^{1 / 2} \Gamma_{i j}^{3}-\frac{\lambda_{i}}{2 g_{i i}} \frac{\partial g_{i i}}{\partial x^{j}},
\end{aligned}
$$

where there is no sum on the repeated index $i$. Equations (43) form a system of 27 first order PDE's in nine independent variables $u_{i}, v_{i}, w_{i}$. This system of equations is overdetermined and thus its solvability should depend on certain compatibility conditions. According to a theorem on the overdetermined system of equations [10], if the compatibility conditions hold then the solution of Eqs. (43) exists and is unique. The conditions

$$
\partial^{2} \lambda_{i} / \partial x^{m} \partial x^{j}=\partial^{2} \lambda_{i} / \partial x^{j} \partial x^{m}
$$

for all values of $i, m$, and $j$ are the compatibility conditions. To prove (44) we use Eq. (A.11), which on cross differentiation yields

$$
\frac{\partial^{2} \mathbf{a}_{i}}{\partial x^{m} \partial x^{j}}-\frac{\partial^{2} \mathbf{a}_{i}}{\partial x^{j} \partial x^{m}}=R_{\cdot i m j}^{l} \mathbf{a}_{l},
$$

where $R_{{ }^{\prime}{ }^{\prime} j}$ is the Riemann-Christoffel curvature tensor and is related to the Riemann's tensor $R_{i j k l}$. Evidently in our present case $R_{{ }^{\prime} i m j}^{l}=0$, since the space is Euclidean. Inserting (40) in (45) we find that Eq. (44) are identically satisfied.

It is interesting to note that for a two-dimensional curvilinear coordinate system there is no need to solve the system of equations such as (43). In this case the single differential equation with $G_{3}=g$

$$
R_{1212}=\sqrt{g}\left[\frac{\partial}{\partial \eta}\left(\frac{\sqrt{g} \Gamma_{11}^{2}}{g_{11}}\right)-\frac{\partial}{\partial \xi}\left(\frac{\sqrt{g} \Gamma_{12}^{2}}{g_{11}}\right)\right]=0
$$

implies the existence of a single function $\alpha(\xi, \eta)$ such that

$$
\alpha_{\xi}=\frac{-\sqrt{g}}{g_{11}} \Gamma_{11}^{2}, \quad \alpha_{\eta}=\frac{-\sqrt{g}}{g_{11}} \Gamma_{12}^{2} .
$$

Consequently

$$
u_{1}=\cos \alpha, \quad v_{1}=-\sin \alpha, \quad u_{2}=\cos (\alpha-\theta), \quad v_{2}=-\sin (\alpha-\theta),
$$

where $\alpha$ is the angle made by the tangent to the coordinate line $\eta=$ const. in a clockwise sense with the $x$-axis, and

$$
\cos \theta=g_{12} / \sqrt{g_{11} g_{22}}
$$

is known. The angle $\alpha$ becomes known since $g_{i j}$ are known; e.g. [9].

3.1 Case of orthogonal coordinates. For orthogonal coordinates since the cosines of the angles between the coordinate curves are zero, we have

$$
g_{12}=g_{13}=g_{23}=0 \text {. }
$$


Consequently,

$$
[12,3]=[13,2]=[23,1]=0, \quad \Gamma_{12}^{3}=\Gamma_{13}^{2}=\Gamma_{23}^{1}=0, \quad g=g_{11} g_{22} g_{33} .
$$

The equations for the metric coefficients, viz. Eqs. (39) under the constraints of orthogonality (46) simply reduce to the Lame's equations. They can concisely be written as six equations by dropping the summation convention in the form

$$
\begin{gathered}
\frac{\partial}{\partial x^{j}}\left(\frac{1}{h_{j}} \frac{\partial h_{k}}{\partial x^{j}}\right)+\frac{\partial}{\partial x^{k}}\left(\frac{1}{h_{k}} \frac{\partial h_{j}}{\partial x^{k}}\right)+\frac{1}{h_{i}^{2}} \frac{\partial h_{j}}{\partial x^{i}} \frac{\partial h_{k}}{\partial x^{i}}=0, \\
\frac{\partial^{2} h_{i}}{\partial x^{j} \partial x^{k}}=\frac{1}{h_{j}} \frac{\partial h_{i}}{\partial x^{j}} \frac{\partial h_{j}}{\partial x^{k}}+\frac{1}{h_{k}} \frac{\partial h_{i}}{\partial x^{k}} \frac{\partial h_{k}}{\partial x^{j}},
\end{gathered}
$$

where $(i, j, k)$ are to be taken in the cyclic permutations of $(1,2,3)$, in this order, and

$$
h_{1}=\sqrt{g_{11}}, \quad h_{2}=\sqrt{g_{22}}, \quad h_{3}=\sqrt{g_{33}} .
$$

To obtain the differential equations for the Cartesian coordinates $x_{1}=x, x_{2}=y$, $x_{3}=z$, we first proceed from Eq. (A.13) and have

$$
\sqrt{g} \nabla^{2} \xi=\frac{\partial}{\partial \xi}\left(h_{2} h_{3} / h_{1}\right), \quad \sqrt{g} \nabla^{2} \eta=\frac{\partial}{\partial \eta}\left(h_{1} h_{3} / h_{2}\right), \quad \sqrt{g} \nabla^{2} \zeta=\frac{\partial}{\partial \zeta}\left(h_{1} h_{2} / h_{3}\right),
$$

where

$$
\sqrt{g}=h_{1} h_{2} h_{3}, \quad \nabla^{2}=\partial_{x x}+\partial_{y y}+\partial_{z z} .
$$

Proceeding directly from Eq. (A.14) and using Eqs. (46) and (48), the equations for the Cartesian coordinates are

$$
\Xi x_{m}=0, \quad m=1,2,3,
$$

where

$$
\Xi=\frac{\partial}{\partial \xi}\left(\frac{h_{2} h_{3}}{h_{1}} \frac{\partial}{\partial \xi}\right)+\frac{\partial}{\partial \eta}\left(\frac{h_{1} h_{3}}{h_{2}} \frac{\partial}{\partial \eta}\right)+\frac{\partial}{\partial \zeta}\left(\frac{h_{1} h_{2}}{h_{3}} \frac{\partial}{\partial \zeta}\right)
$$

Note that the operator $\Xi$ and the Laplacian $\nabla^{2}$ are related as

$$
\Xi \phi=h_{1} h_{2} h_{3} \nabla^{2} \phi
$$

where $\phi$ is a scalar.

Equations (47) and (49) are those consistent set of equations which every orthogonal coordinate system must satisfy.

3.2 The case of isothermic coordinates. Isothermic coordinates in a surface embedded in a $3 D$ Euclidean space are those coordinates in which the metric coefficients $g_{11}$ and $g_{33}$ in the surface $\eta=$ const. are equal. That is, the element of length $d s$ on $\eta=$ const. is given by

$$
(d s)_{\eta=\text { const. }}^{2}=g_{11}\left[(d \xi)^{2}+(d \zeta)^{2}\right]
$$

where $\xi, \zeta$ are chosen to be the surface coordinates. Using (46) and setting

$$
g_{33}=g_{11} \text { and } g_{22}=F(\eta)
$$


in Eqs. (39), we obtain the basic equations for $g_{11}$, which are

$$
\begin{aligned}
\frac{\partial}{\partial \xi}\left(\frac{1}{g_{11}} \frac{\partial g_{11}}{\partial \xi}\right)+\frac{\partial}{\partial \zeta}\left(\frac{1}{g_{11}} \frac{\partial g_{11}}{\partial \zeta}\right)+\frac{1}{2 F g_{11}}\left(\frac{\partial g_{11}}{\partial \eta}\right)^{2}=0, \\
\frac{\partial}{\partial \eta}\left(\frac{1}{\sqrt{F g_{11}}} \frac{\partial g_{11}}{\partial \eta}\right)=0, \\
\frac{\partial}{\partial \zeta}\left(\frac{1}{g_{11}} \frac{\partial g_{11}}{\partial \eta}\right)=0, \\
\frac{\partial}{\partial \xi}\left(\frac{1}{g_{11}} \frac{\partial g_{11}}{\partial \eta}\right)=0 .
\end{aligned}
$$

It can easily be verified that the only solution of Eqs. $(50 c, d)$ is

$$
g_{11}=[a+P(\eta)]^{2} f(\xi, \zeta), \quad a=\text { const. }
$$

Thus from $(50 \mathrm{~b})$

$$
F(\eta)=(d P / d \eta)^{2} .
$$

Substituting (51) and (52) in Eq. (50a), the differential equation for $f(\xi, \zeta)$ becomes

$$
\frac{\partial}{\partial \xi}\left(\frac{1}{f} \frac{\partial f}{\partial \xi}\right)+\frac{\partial}{\partial \zeta}\left(\frac{1}{f} \frac{\partial f}{\partial \zeta}\right)+2 f=0 .
$$

In Kreyszig [11], we have the result that if in a portion of a surface isothermic coordinates can be introduced then that portion of the surface can conformally be mapped onto a plane. Thus in effect the solution of Eq. (53) provides that mapping function which conformally maps a surface onto a plane. As a verification of the above conclusion, we verify that the function

$$
f=4 e^{25} /\left(1+e^{25}\right)^{2}
$$

is a solution of Eq. (53). This function is related with the isothermic coordinates on a sphere. Using the parametric equations of a sphere

$$
x=[a+P(\eta)] \cos \theta, \quad y=[a+P(\eta)] \sin \theta \sin \phi, \quad z=[a+P(\eta)] \sin \theta \cos \phi
$$

and writing

$$
\xi=\phi, \quad \zeta=\ln \tan \frac{\theta}{2},
$$

where $0<\phi<2 \pi$ and $0<\theta<\pi$, we obtain

$$
g_{33}=g_{11}=4(a+P)^{2} e^{2 \zeta} /\left(1+e^{2 \zeta}\right)^{2} .
$$

Thus the equations

$$
\begin{aligned}
& x=(a+P)\left(1-e^{2 \zeta}\right) /\left(1+e^{2 \zeta}\right), \\
& y=2(a+P) e^{\zeta} \sin \xi /\left(1+e^{2 \zeta}\right), \\
& z=2(a+P) e^{\zeta} \cos \xi /\left(1+e^{2 \zeta}\right)
\end{aligned}
$$


represent a sphere of radius $a+P(\eta)$ in terms of the isothermic coordinates $\xi, \zeta$ in the surface. Since $P(\eta)$ is an arbitrary function of $\eta$, we now have the capability of prescribing a suitable function $P(\eta)$ to achieve any sort of contraction on expansion in the field. It is expected that the representation (55) should prove useful in the computational problems associated with a sphere.

4. Conclusions. In Sec. 2 of this paper a set of second order PDE's have been obtained which generate a series of surfaces between the given inner and outer arbitrary shaped bodies. The necessary mathematical apparatus which connects one generated surface with its neighbor along with the curvature properties of each surface has been incorporated in the right hand side terms of the equations. (Eqs. (20) or (22)). By changing the computational techniques these equations can also be used to generate the $3 D$ coordinates when more than one inner bodies are present in the field.

In Sec. 3, based on some basic differential geometric concepts, a number of field equations have been isolated which must always be satisfied by any coordinate system in an Euclidean space. Efficient numerical methods are to be developed to solve these quasilinear equations (Eqs. (39)) on a digital computer.

Appendix A. In this appendix we collect some useful formulae which have been used in the main text.

As noted in the text, a general curvilinear coordinate system is denoted as $x^{i}, i=1,2,3$, or as $\xi, \eta, \zeta$, while a rectangular Cartesian system is denoted as $x_{m}, m=1,2,3$ or as $x, y, z$. Since $\mathbf{r}$ is a position vector in an Euclidean space, the covariant base vectors $\mathbf{a}_{i}$ are given by

$$
\mathbf{a}_{i}=\partial \mathbf{r} / \partial x^{i}
$$

while the contravariant base vectors $\mathbf{a}^{i}$ are given by

$$
\mathbf{a}^{i}=\operatorname{grad} x^{i} .
$$

The covariant and the contravariant metric components are respectively given by

$$
g_{i j}=\mathbf{a}_{i} \cdot \mathbf{a}_{j}, \quad g^{i j}=\mathbf{a}^{i} \cdot \mathbf{a}^{j} .
$$

Both metric coefficients are related through the equations

$$
g^{i j} g_{i k}=\delta_{k}^{j},
$$

where $\delta k$ are the Kronecker deltas. Also

$$
g=\operatorname{det}\left(g^{i j}\right), \quad g \hat{g}=1 .
$$

Based on (A.4), we also have

$$
\begin{aligned}
\mathbf{a}^{i} & =g^{i j} \mathbf{a}_{j} \\
& =e^{i j k}\left(\mathbf{a}_{j} \times \mathbf{a}_{k}\right) / 2 \sqrt{g},
\end{aligned}
$$

where, here and in all the expressions a repeated lower and upper index always stands for a sum over the range of index values. Also 


$$
\begin{aligned}
g & =\operatorname{det}\left(g_{i j}\right)=g_{33} G_{3}+g_{13} G_{5}+g_{23} G_{6} \\
& =g_{22} G_{2}+g_{12} G_{4}+g_{23} G_{6}=g_{11} G_{1}+g_{12} G_{4}+g_{13} G_{5},
\end{aligned}
$$

where

$$
\begin{array}{lll}
G_{1}=g_{22} g_{33}-\left(g_{23}\right)^{2}, & G_{2}=g_{11} g_{33}-\left(g_{13}\right)^{2}, & G_{3}=g_{11} g_{22}-\left(g_{12}\right)^{2}, \\
G_{4}=g_{13} g_{23}-g_{12} g_{33}, & G_{5}=g_{12} g_{23}-g_{13} g_{22}, & G_{6}=g_{12} g_{13}-g_{23} g_{11} .
\end{array}
$$

Note that

$$
\begin{array}{lll}
g^{11}=G_{1} / g, & g^{22}=G_{2} / g, & g^{33}=G_{3} / g, \\
g^{12}=G_{4} / g, & g^{13}=G_{5} / g, & g^{23}=G_{6} / g .
\end{array}
$$

The derivative of a covariant base vector is given by

$$
\partial \mathbf{a}_{i} / \partial x^{j}=\partial^{2} \mathbf{r} / \partial x^{i} \partial x^{j}=\Gamma_{i j}^{l} \mathbf{a}_{l} .
$$

The Laplacian of a scalar $\phi$ in a curvilinear coordinate system is

$$
\nabla^{2} \phi=g^{i j}\left(\frac{\partial^{2} \phi}{\partial x^{i} \partial x^{j}}-\Gamma_{i j}^{r} \frac{\partial \phi}{\partial x^{r}}\right)
$$

where $\Gamma_{i j}^{r}$ have been defined in Eqs. (6).

If $\phi=x^{m}$ is any curvilinear coordinate then from (A.12)

$$
\nabla^{2} x^{m}=-g^{i j} \Gamma_{i j}^{m}
$$

If $\phi=x_{m}$ is any rectangular Cartesian coordinate then from (A.12)

$$
g^{i j} \frac{\partial^{2} x_{m}}{\partial x^{i} \partial x^{j}}+\left(\nabla^{2} x^{r}\right) \frac{\partial x_{m}}{\partial x^{r}}=0
$$

Appendix B. In numerical computations it is desirable to solve Eqs. (22) in their limiting forms on certain special lines in the field. Referring to Fig. (2a), let the $x$-axis be aligned to pass through the inner body from two of its points, which, when extended in both directions meets the outer body at its two corresponding points. The portions of the lines between the inner and the outer bodies form the right and the left segments. On each segment $y=z=0$, and according to the adopted convention $\zeta=0$ and $\zeta=\pi$ on the right and the left segments respectively for all values of $\xi$. With this choice of the axes only Eq. (22a) is of interest. Taking the limit of Eq. (22a) as $\zeta \rightarrow 0$ or $\zeta \rightarrow \pi$, we obtain

$$
x_{\bar{\eta} \bar{\eta}}+P_{22}^{2} x_{\bar{\eta}}=\operatorname{Lim}_{\substack{\zeta \rightarrow 0 \\ \zeta \rightarrow \pi}}\left(\bar{x} \bar{\lambda} \bar{g}_{22} \bar{\Gamma}_{11}^{3} / \bar{g}_{11}\right) .
$$

where the control function $P_{22}^{2}$ has already been chosen a priori. The terms on the right hand side of Eq. (B.1) are difficult to assess for their limiting behaviors. However, some guidance can be obtained from the exact solution discussed in Sec. 2.3. This approach suggests that in any case, the following estimates can be used. 


$$
\begin{aligned}
\bar{X} & =f_{1}(\bar{\eta})\left(\bar{g}_{11}\right)^{1 / 2} / x_{\eta}, \quad \bar{\lambda}=f_{2}(\bar{\eta}), \\
\bar{\Gamma}_{11}^{3} & =f_{3}(\bar{\eta})\left(\bar{g}_{11}\right)^{1 / 2}, \quad \bar{g}_{22}=x_{\bar{\eta}}^{2}, \quad \text { for } \zeta \rightarrow 0 \text { or } \pi,
\end{aligned}
$$

where $f_{1}, f_{2}, f_{3}$ are functions of $\bar{\eta}$. Using the estimates (B.2) in Eq. (B.1), we obtain

$$
x_{\bar{\eta} \bar{\eta}}+T(\bar{\eta}) x_{\bar{\eta}}=0,
$$

where

$$
T(\bar{\eta})=P_{22}^{2}-F(\bar{\eta}), \quad F(\bar{\eta})=f_{1} f_{2} f_{3} .
$$

The scheme now is to solve Eq. (B.3) by prescribing $T(\bar{\eta}) \neq P_{22}^{2}$ arbitrarily to achieve the desired control of points on the segments. Since $P_{22}^{2}$ has already been chosen in advance this approach produces those values of $F(\bar{\eta})$ (though they need not be calculated) which are consistent with the basic equation, viz., Eq. (22a).

\section{REFERENCES}

[1] A. M. Winslow, Numerical solution of the quasi-linear Poisson equation in a non-uniform triangular mesh, J. Comp. Phys. 2, 149-172 (1967)

[2] J. F. Thompson, F. C. Thames, and C. W. Mastin, Automatic numerical generation of body-fitted curvilinear coordinate system for field containing any number of arbitrary two-dimensional bodies, J. Comp. Phys. 15, 299-319 (1974)

[3] J. F. Thompson, Z. U. A. Warsi, and C. W. Mastin, Boundary-fitted coordinate systems for numerical solution of partial differential equations - a review, J. Comp. Phys. 47, 1-108 (1982)

[4] Z. U. A. Warsi, A method for the generation of general three-dimensional coordinates between bodies of arbitrary shapes, Engineering and Industrial Research Station, Mississippi State University, Rept. MSSUEIRS-80-7 (1980)

[5] expm, Tensors and differential geometry applied to analytic and numerical coordinate generation, Engineering and Industrial Research Station, Mississippi State University, Rept. MSSU-EIRS-81-1 (1981)

[6] expm, Basic differential models for coordinate generation, Symposium on the Numerical Generation of Curvilinear Coordinate Systems and Use in the Numerical Solution of Partial Differential Equations, organized by Mississippi State University, sponsored by NASA and AFOSR. Radisson Plaza, Nashville, Tennessee, April 13-16 (1982); Appl. Math. Comp. 10, 41-77 (1982)

[7] Z. U. A. Warsi and J. P. Ziebarth, Numerical generation of three-dimensional coordinates between bodies of arbitrary shapes, Ibid. pp. 717-728

[8] L. P. Eisenhart, An introduction to differential geometry with use of the tensor calculus, Princeton University Press, Princeton, N.J., 1947

[9] Z. U. A. Warsi and J. F. Thompson, A noniterative method for the generation of orthogonal coordinates in doubly-connected regions, Math. Comp. 38, 501-516 (1982)

[10] J. J. Stoker, Differential geometry, Wiley-Interscience, New York, 1969

[11] E. Kreyszig, Differential geometry, University of Toronto Press, Toronto, 1959 